\title{
Where's the difficulty in standardized reading tests: The passage or the question?
}

\author{
Yasuhiro Ozuru and Michael Rowe \\ University of Memphis, Memphis, Tennessee \\ Tenaha O'ReILly \\ Educational Testing Service, Princeton, New Jersey \\ AND \\ Danielle S. MCNamara \\ University of Memphis, Memphis, Tennessee
}

\begin{abstract}
The purpose of this investigation was to examine the extent to which item and text characteristics predict item difficulty on the comprehension portion of the Gates-MacGinitie Reading Tests for the 7th-9th and 10th-12th grade levels. Detailed item-based analyses were performed on 192 comprehension questions on the basis of the cognitive processing model framework proposed by Embretson and colleagues (Embretson \& Wetzel, 1987). Item difficulty was analyzed in terms of various passage features (e.g., word frequency and number of propositions) and individual-question characteristics (e.g., abstractness and degree of inferential processing), using hierarchical linear modeling. The results indicated that the difficulty of the items in the test for the 7th-9th grade level is primarily influenced by text features - in particular, vocabulary difficulty - whereas the difficulty of the items in the test for the 10th-12th grade level is less systematically influenced by text features.
\end{abstract}

Students' reading comprehension ability is frequently assessed in school settings, using some form of standardized reading comprehension ability test. In a typical standardized reading comprehension ability test-in particular, commercial standardized tests - test takers read a variety of short passages and answer multiple-choice questions about the passage content (e.gm the Gates-MacGinitie Reading Comprehension Ability Test, the Nelson-Denny Reading Ability Test, and the Scholastic Aptitude Test [SAT]). This type of standardized reading test is assumed to measure the components of reading ability, because performing well on the test requires reading various types of passages and correctly answering questions about the passages. These types of tests are often favored by educators and researchers because they tend to have relatively high reliability. However, some concerns have been raised concerning the validity of standardized reading ability tests and whether they are able to diagnose readers' specific abilities and deficits (e.g., Daneman \& Hannon, 2001; Magliano, Millis, Ozuru, \& McNamara, 2007; VanderVeen et al., 2007). Hence, in this study, we sought to more thoroughly examine the source of difficulty for readers in one such standardized test, the Gates-MacGinitie Reading Test (GMRT, 1989; MacGinitie \& McGinitie, 1989). We present the results of detailed item-based analyses guided by cognitive theories of text processing. These analyses were performed on the reading comprehension portion of two sets of the GMRT - specifically, the 7th-9th grade level and the 10th-12th grade level tests.

\section{Reading Comprehension Assessment}

There exist at least two significant challenges in designing effective methods to assess reading comprehension (Fletcher, 2006; Pearson \& Hamm, 2005; Snow, 2003). The first major challenge is that reading comprehension is a complex and multicomponent process (Fletcher, 2006; Hannon \& Daneman, 2001; Snow, 2003). That is, in order to successfully comprehend written materials, readers need to successfully and fluently execute various cognitive processes that may draw upon different resources. These processes or subprocesses include the following: the phonological decoding of written words (Perfetti, Beck, Bell, \& Hughes, 1987); accessing the meanings of individual words (Snow, Barnes, Chandler, Goodman, \& Hemphill, 1991); combining the individual-word meanings to form a coherent sentence meaning, using syntactic knowledge (Marslen-Wilson, 1975); and integrating individualsentence meanings with larger discourse-level meanings, using language-specific knowledge and/or general knowledge (e.g., world knowledge or domain knowledge; Adams \& Collins, 1979; Kintsch, 1988, 1998). Thus, reading comprehension problems and/or variability in reading

Y.Ozuru, yasozuru@uic.edu 
comprehension ability arise for many reasons, such as differences in competencies associated with any single subprocess or combination of underlying subprocesses.

The second major challenge to measuring reading comprehension involves the variability in reading comprehension situations. People read for different purposes, including reading popular magazines or novels for entertainment, reading a science text to learn new knowledge at school, reading a poem and analyzing the author's intention in order to write a critical essay, or reading multiple texts to compare and synthesize information (Wolfe \& Goldman, 2005). These varied reading comprehension situations are likely to call for different types of comprehension processes, abilities, or strategies (van den Broek, Lorch, Linderholm, \& Gustafson, 2001). The diversity in reading comprehension situations, combined with the multicomponent nature of reading comprehension ability, contributes to the challenges faced in reading comprehension assessment.

Messick (1989, 1996a, 1996b) has addressed a way in which the complexity and multifaceted nature of validity in the testing of complex psychological constructs, such as reading comprehension ability, should be confronted. Messick highlighted the importance of addressing six aspects of validity: content, substantive aspects, structural aspects, generalizability, external factors, and consequential aspects of validity. He further argued that a heavy emphasis should be placed on the practical aspects of validity (i.e., the consequential aspects of validity), particularly with respect to how scores on the measurement can be used to make decisions in social and educational contexts. Specifically, he argued that it is important to consider what practical purpose an assessment serves, given the strengths and weaknesses of the tool as a whole. More generally, and of importance here, he argued that a clearer understanding of the strengths and weaknesses of psychological and educational assessments can be achieved only by considering various aspects of validity.

In line with Messick's (1989, 1996a, 1996b) proposals on the validity of psychological assessment, research on reading comprehension assessments, particularly on standardized reading comprehension assessment, has examined various issues with regard to validity (for reviews, see Campbell, 1999; Pearson \& Hamm, 2005). For example, some researchers have pointed to the artificial nature of existing reading comprehension assessments, questioning the generalizability of standardized reading comprehension tests. Many traditional reading comprehension assessments consist of passages that are made deliberately brief in order to increase the total number of items in the assessment and to cover a wider range of topics. This wider coverage is intended to reduce the effects of prior knowledge on performance (e.g., the Nelson-Denny and Gates-MacGinitie tests). In addition, as compared with texts that are routinely read in classrooms, the structure of texts used in reading comprehension assessments tends to be less apparent, due to their brevity (Sternberg, 1991; Valencia \& Pearson, 1988).

Research on the validity of assessment tools has also investigated question format - in particular, the use of multiple-choice questions. Multiple-choice questions are considered problematic for a number of reasons. For instance, Katz, Blackburn, and Lautenschlager (1991) found that students can perform at above-chance levels on multiple-choice questions on the SAT without reading the passage associated with the questions. This finding raised the question of whether multiple-choice questions are valid, and what measures of reading comprehension ability should be used.

Another concern is that multiple-choice questions force students to select predetermined answers, when other, more viable options may exist. Consequently, multiplechoice comprehension questions can result in test performances that may not accurately reflect students' ability to construct meaning from texts (Valencia \& Pearson, 1988). Motivated by these concerns, researchers have used both statistical and experimental approaches to examine differences between multiple-choice and open-ended comprehension assessments in terms of their ability to accurately measure reading comprehension.

One statistical approach is to perform a factor analysis to examine the amount of common variance in reading comprehension shared by multiple-choice and openended format questions. For example, Bridgeman and Rock (1993) performed a factor analysis on the Graduate Record Exam (GRE) general test scores and found no significant differences between the two formats with respect to their ability to measure factors underlying the reading comprehension processes. On the other hand, Manhart's (1996) factor analysis on multiple-choice and constructedresponse science tests revealed a significant difference between the two formats, suggesting that multiple-choice and open-ended questions may measure slightly different aspects or components of reading comprehension.

Researchers with an experimentally oriented approach (e.g., Campbell, 1999; Cordon \& Day, 1996; Pearson et al., 1999) have investigated the effect of item format on the validity of multiple-choice comprehension assessments, using think-aloud procedures (Ericsson \& Simon, 1993). In this type of research, the readers verbalize their thoughts while answering multiple-choice or open-ended questions. The contents of the verbal protocols are analyzed to examine the presence of possible differences in the answering process. Some studies have failed to detect differences in the cognitive processes underlying openended and multiple-choice comprehension question answering (e.g., Campbell, 1999; Cordon \& Day, 1996). However, Pearson et al. found that multiple-choice questions were less likely than open-ended questions to elicit multiple strategies and intertextual strategies.

Recent research on reading comprehension assessment has also incorporated cognitive psychology research on text processing and comprehension (Embretson, 1998; Mislevy, 1994, 1995). This approach decomposes a task (i.e., taking a comprehension test) into a processing model (Embretson, 1998) and then examines the contribution of particular processes (or subprocesses) to item responses. Following this approach, a theory-driven detailed analysis is performed on the test items used in a reading comprehension assessment. The results of this analysis potentially 
help to identify which types of item features (e.g., type of questions, passage length, etc.) contribute most to the difficulty level of the items. This information, in conjunction with a cognitive theory of text processing and comprehension, can afford researchers and educators greater insight into the types of cognitive processing (e.g., vocabulary knowledge or syntactic processing) that tend to be tapped by the items included in the assessment.

Several studies based on this approach were conducted by Embretson and colleagues (Embretson \& Wetzel, 1987; Gorin, Embretson, \& Sheehan, 2002). Embretson and Wetzel analyzed question items contained in several reading comprehension tests (e.g., the Army Services Vocational Aptitude Battery and the GRE) and showed that features of the passage and question items were related to item difficulty. They found that the propositional density of the passage and the extensiveness of the reasoning required to map the question and answer onto the passage were the two main factors that influenced item difficulty. A similar analysis was performed by Sheehan and Ginther (2001) on reading comprehension questions from the Test of English as a Foreign Language (TOEFL). They found that item difficulty was largely influenced by the frequency and location of the target information. Overall, these studies have collectively shown that this type of theory-based analysis of test items provides useful information about the variability in test takers' reading ability as measured by these tests.

\section{The Present Study}

Following the cognitive processing model framework proposed by Embretson and Wetzel (1987), we conducted item-based analyses of 192 comprehension questions from the 7 th -9 th and 10th-12th grade levels of the GMRT. The GMRT is a widely used standardized test of reading ability. We analyzed the difficulty level of each item (i.e., the proportion of test takers in a standardization sample who provided the correct answer to an item according to the test publisher), using various passage features and individual-question characteristics as predictor variables. This analysis involved predicting the difficulty level of items, with individual-item characteristics (i.e., features of questions) and passage features as predictor variables. When using passage features as predictor variables, we selected several features that are known to affect reading comprehension, including the number of propositions in a passage (i.e., propositional density), the number of words per sentence, and word frequency. Propositional density is known to affect the overall information-processing demands of a text (Kintsch \& van Dijk, 1978) and item difficulty (Embretson \& Wetzel, 1987). Similarly, processing a longer sentence places greater demands on working memory (Just \& Carpenter, 1992). So, passages with sentences that contain more words are potentially more difficult to comprehend. In addition, when a passage contains unfamiliar, infrequent words, readers often experience difficulty in understanding the text, resulting in lower performance on the questions associated with the passage (Perfetti, 1985).
The item characteristics of individual questions also influence an item's difficulty. To assess these characteristics, we included two item features that were predicted to influence item difficulty on the basis of Embretson and Wetzel's (1987) first study: (1) the type of comprehension process required to answer the question and (2) the number of explicitly falsifiable distractors. In addition, we also used Mosenthal's (1996) coding scheme for abstractness of the requested information-specifically, the level of abstractness in the information requested by the question and target answer. The rationale behind this coding scheme is that searching for abstract, as opposed to concrete, information in a passage tends to require a more extensive search and more information integration, rendering the task more difficult (Mosenthal, 1996).

The goal of this study was to provide a detailed analysis of the GMRT 7th-9th and 10th-12th grade level reading tests. The reason that we analyzed two levels of the tests, rather than only one, was to explore the possibility that reading ability tests targeting different grade levels represent different aspects of reading ability (i.e., lower levels and higher levels). That is, these two tests may and should differentiate students' reading ability on the basis of different component processes (e.g., vocabulary vs. inferential ability).

We analyzed the contribution of item and passage features to each question's difficulty level for both levels of the test (7th-9th and 10th-12th grades) separately, using hierarchical linear modeling (HLM). HLM is a powerful statistical technique that differentiates the contribution of multiple factors located at multiple levels of a nested data structure (e.g., comprehension questions nested across multiple passages; see Richter, 2006, for a review of HLM in psychological research on text comprehension). It should be noted that the effects of passage features and item features found in this HLM analysis generalize beyond the specific reading comprehension questions analyzed in this research, provided that the test takers are comparable, because individual items are treated as a random factor in the analysis. The HLM analyses were complemented by the comparison of individual-item and passage features between the two levels of the test and the qualitative observations of the test materials.

\section{METHOD}

\section{Materials}

A total of 192 multiple-choice questions ( 96 from the comprehension portion of the GMRT, third edition, for the 7th-9th grade level, Forms K and L, and 96 from the GMRT, fourth edition, for the 10th12th grade level, Forms $\mathrm{S}$ and T) were the targets of this analysis. The GMRT technical report stated that the third and fourth editions are highly correlated. The 96 questions for the 7th-9th grade level (48 items each for Forms $\mathrm{K}$ and $\mathrm{L}$ ) were based on 28 passages (14 each for Forms K and L). The 96 questions for the 10th-12th grade level (48 items each for Forms $\mathrm{S}$ and T) were based on 22 passages (11 passages each for Forms $\mathrm{S}$ and T). The technical report for the GMRT third edition (Gates-MacGinitie Reading Tests, 1989) and fourth edition provided item difficulty levels in terms of the proportion of test takers in the standardization sample who answered the item correctly. 
The present study differed from the Embretson and Wetzel (1987) study in terms of the variable used to represent the difficulty level of individual items in the test. Whereas Embretson and Wetzel used an item difficulty parameter based on item response theory, in the present study, item difficulty was based on classical test theory. In item response theory, item difficulty is derived from a model-based analysis in which the absolute level of the item's difficulty is extracted. Hence, it is capable of representing the difficulty level of an item independently of the test taker's ability level (Embretson \& Reise, 2000). On the other hand, item difficulty scores used in the present study, based on classical test theory, were specific only to this sample, because they represented the proportion of test takers in the sample who correctly answered a question.

This difference gives rise to two concerns: (1) the influence of the ability of the individual test taker in the sample on the item difficulty score and (2) the mortality of test takers across items. With respect to the effect of sample on the score, the item's difficulty used in this analysis was based on the standardization sample. Hence, the item difficulty score should reflect the item's difficulty level among a representative sample of the target population for the test.

The second concern emanated from the notion that item difficulty (proportion correct) is likely to be affected by the position of the items in the test (i.e., beginning vs. end). That is, only relatively higher ability readers, with a sufficiently high response speed, are likely to produce responses to items located toward the end of the test. Thus, it is possible that the difficulty level of items located toward the end might be inaccurate because a smaller number of test takers attempted to answer these items and those who completed the items were more skilled readers. This concern was at least partly alleviated on the basis of a statement in the GMRT Technical Report that the GMRT is a power test and that more than $85 \%$ of the students who took the test completed it and at least $95 \%$ of the students completed three quarters of the questions. Thus, bias based on mortality is likely to be negligible.

The technical report separately listed item difficulty for different grades of children for fall and spring semesters. We used the item difficulty corresponding to 9th grade children in the spring semester for the analysis of the GMRT 7th-9th grade level and to 12th grade children in the spring semester for the analyses of the GMRT 10th-12th grade level. The first two practice questions for each form were excluded from the analysis. Two coders (the first and second authors) reviewed together the first half of the items in the GMRT 7th-9th grade level, Form K, to familiarize themselves with the coding scheme and the materials. During this process, the coders discussed recurrent discrepancies and problems, until they became reasonably comfortable with their level of agreement. Subsequently, they coded $15 \%$ of total question items from three different texts within the GMRT 7th-9th grade level. The two raters agreed on at least $80 \%$ of their codings on the basis of the two dimensions of the item coding: type of comprehension process required to answer the question and abstractness of the requested information. However, the coders' agreement on the number of distractor options explicitly falsifiable on the basis of the source passage was low (below 50\%). Our solution to this problem was to dichotomize this coding (i.e., any distractor option is falsifiable, instead of specifying the number of distractors falsifiable). A dichotomized version of the coding achieved a satisfactory level of agreement (80\%). After verifying reliability, all of the items and passages (including the ones used in training) were coded by both of the coders together.

\section{Coding of Individual Questions}

The 192 individual questions and their answer options were coded in terms of their relationship with the target passage, using three different classification schemes. The first coding classified the questions in terms of relations between passage and question (passage/ question relations). This coding system addressed the type of passage comprehension processes that test takers needed to engage, in order to answer the question correctly. There were four levels in this scheme. The first level comprised text-based questions, for which the answer to the question was explicitly stated within a single sentence in almost verbatim fashion, so that minimal text processing was required. The second level involved restructuring/rewording within a sentence. Answering this type of question involved identifying a sentence in the source passage in which the target information for the question was reworded or restructured. As for the first level, the information required to answer this question was within a single sentence. The third level comprised integration or bridging questions. Answering this type of question required some degree of integration of information located across multiple sentences from the source passage. Finally, the fourth level was knowledge-based inference questions. In this type of question, the information required to answer a question was not explicitly stated in the source passage. The test takers had to make inferences about the situation described in the passage on the basis of their prior knowledge.

This coding scheme was somewhat similar to Anderson's (1972) rating system on "transformation required to answer questions," used by Embretson and Wetzel (1987). We used this coding scheme, as opposed to Anderson's scheme, for two reasons. First, relative to Anderson's scheme, our coding scheme was more closely built on the basis of a well-known theory of text comprehension (i.e., the construction integration model; Kintsch, 1988, 1998). Specifically, our coding scheme differentiated shallow comprehension of a sentence (comprehension of information explicitly stated within a sentence), deeper level comprehension (restructuring and/or rewording) of a sentence, and two different aspects of further deeper level comprehension of more extended text (across-sentence integration of information and integration of textual information with prior knowledge). Second, we could expect more reliable coding on the basis of this system because a similar coding scheme had been used in our laboratory to generate and classify text comprehension questions in a number of studies (e.g., Ozuru, Best, Bell, Witherspoon, $\&$ McNamara, 2007) and, thus, the coders were already familiar with this scheme.

The second coding system involved the abstractness of the information requested by a question, using Mosenthal's (1996) scheme. This coding scheme addressed the level of abstractness or concreteness of the information requested by the question. Abstractness of the item was classified into five levels. The first level, most concrete, asked for the "identification of persons, animals, or things." The second level, the highly concrete class of questions, asked for the "identification of amounts, times, or attributes." The third level, intermediate questions, asked for the "identification of manner, goal, purpose, alternative, attempt, or condition." The fourth level, highly abstract, asked for the "identification of cause, effect, reason, or result." The highest level, the most abstract questions, asked for the "identification of equivalence, difference, or theme."

In addition to the previously mentioned two schemes that analyzed question stem and correct answer together in relation to the passage, we also coded the quality of distractor options, following the coding method of Embretson and Wetzel (1987). This analysis addressed the number of distractors that could have been explicitly falsified by the content of the passage. A distractor was falsifiable if the passage provided explicit textual evidence that the distractor was incorrect. A distractor was not falsifiable if the passage had no explicit mention of the distractor. However, due to a problem in reaching coding reliability (see above), we used a dichotomized version of the coding in HLM analysis in which the effect of the number of falsifiable distractors on item difficulty was examined. That is, the falsifiability of distractors was classified into two dichotomous categories in the HLM analysis: no distractor was falsifiable versus at least one distractor option was falsifiable. We acknowledge that use of continuous coding (i.e., actual number of falsifiable answer options), as opposed to dichotomous coding (i.e., whether at least one answer option could be falsifiable), may be preferable because having three falsifiable distractors, instead of only one falsifiable distractor, may make an item easier. However, the difficulty involved in reliably counting the number of clearly falsifiable distractors led us to this decision of using the dichotomous coding in the 
HLM analysis. Specifically, it was often difficult to judge whether a given distractor was falsifiable when the requested information was abstract and when the answer required integration of multiple sentences and/or knowledge-based inferences. Nonetheless, we report the number of items with zero, one, two, or three falsifiable distractors in Table 1 for descriptive purposes only.

Example of coding process. The following is an example of the coding processes used for each question in the study. An example question will be presented first, followed by each of the three coding processes. For example, one question asks students to complete a sentence by selecting a word from among four options. An excerpt from the text where the answer resides is provided.

Tom bent over a large lizard, gay in green and orange, and at last put out a small finger to feel the color. The lizard bit him, and his whole arm swelled from the poison.

Tom touched the lizard because it was

A. unusual

B. weak

C. colorful

D. small

With respect to passage question relations, this item would be coded as a restructuring/rewording item because the information required to answer the question (green and orange) is stated within a single sentence, but not in the same way as the target answer (i.e., colorful) in the answer options. With respect to the second coding dimension (i.e., abstractness), the item would be coded as highly concrete, because the to-be-identified information, colorfulness, is a fairly concrete attribute. With respect to the falsifiability of distractor items, one distractor (i.e., small) is falsifiable on the basis of the passage, because the text explicitly states that the lizard is large.

\section{Analyses of Passage Features}

Passage features were analyzed using Coh-Metrix v1.4 (Graesser, McNamara, Louwerse, \& Cai, 2004), a computational tool that assesses 238 cohesion and readability measures considered to influence comprehension. For the present study, we focused on three measures that were theoretically and empirically known to influence comprehension difficulty: number of propositions, number of words per sentence, and the logarithm of the average minimum frequency content word in each sentence (averaging the word frequency of the minimum frequency content word in each sentence across the passage). We also included the Flesch Reading Ease score when presenting the type of passages in the test and the correlations between the passage features, but we did not include Flesch Reading Ease as a predictor variable in HLM, because Flesch Reading Ease is computed on the basis of sentence length and word frequency and these two passage features variables were already included in the analysis. Number of propositions was approximated using the Charniak (2000) parser, which counted the number of clauses, phrases, and parts of speech in a sentence. Clause count was used to approximate the number of propositions in a sentence.

\section{RESULTS AND DISCUSSION}

We will discuss the results of this research in three sections. First, we will report the descriptions of the individualitem features and passage features for the 7th-9th and the 10th-12th grade levels of the GMRT. Second, we will present the results of the analysis examining the contribution of individual-item and passage features on the item's difficulty, using HLM. Third, we will compare the 7th-9th and 10th-12th grade levels on the basis of the results of the HLM, of an item/passage feature-based comparison, and of a qualitative observation of the materials used in the test.

\section{Overall Descriptions of \\ Passage and Item Features}

Table 1 presents the results of item coding based on the three coding schemes used. Both the 7th-9th and the 10th-12th GMRT grade levels contain a variety of item types that vary in terms of three item features: the comprehension processes necessary to answer the question correctly, the abstractness of the information requested, and the falsifiability of the distractor options. The results show that both tests (i.e., 7th-9th and 10th-12th grade levels) are attempts to measure the different levels of reading ability by using a range of questions that require more or less integration of and/or inferencing about information that varies in abstractness. One interesting finding is that across the two test levels, at least one distractor item is explicitly falsifiable for a relatively large number of items.

Table 2 presents the frequencies of passage features in categorical levels (e.g., reading ease and propositional density) for the GMRT 7th-9th and 10th-12th grade

Table 1

Frequency of the Question Items and Their Mean Item Difficulty (With Standard

Deviations) As a Function of Question Characteristics for the

7th-9th and 10th-12th Grade Levels of the Gates-MacGinitie Reading Test

\begin{tabular}{|c|c|c|c|c|c|c|c|}
\hline \multirow{2}{*}{$\begin{array}{c}\text { Classification } \\
\text { Scheme }\end{array}$} & \multirow[b]{2}{*}{ Type of Questions } & \multicolumn{3}{|c|}{7 th-9th } & \multicolumn{3}{|c|}{10 th-12th } \\
\hline & & $n$ & $M$ & $S D$ & $n$ & $M$ & $S D$ \\
\hline \multirow[t]{4}{*}{ Passage/question relations } & Text based & 23 & .67 & .15 & 4 & .66 & .14 \\
\hline & Reword/restructure & 26 & .66 & .14 & 21 & .60 & .13 \\
\hline & Bridging & 17 & .67 & .10 & 17 & .60 & .13 \\
\hline & Inferential & 30 & .67 & .15 & 54 & .62 & .13 \\
\hline \multirow[t]{5}{*}{ Abstractness } & Most concrete & 9 & .65 & .12 & 18 & .63 & .11 \\
\hline & Highly concrete & 34 & .66 & .16 & 32 & .62 & .15 \\
\hline & Intermediate & 18 & .72 & .15 & 6 & .57 & .16 \\
\hline & Highly abstract & 23 & .66 & .16 & 25 & .60 & .14 \\
\hline & Most abstract & 12 & .65 & .17 & 15 & .63 & .07 \\
\hline \multirow[t]{4}{*}{ Number of falsifiable distractors } & None & 43 & .68 & .16 & 57 & .60 & .13 \\
\hline & One & 32 & .64 & .15 & 29 & .64 & .14 \\
\hline & Two & 18 & .69 & .15 & 5 & .62 & .09 \\
\hline & Three & 3 & .76 & .13 & 5 & .62 & .06 \\
\hline
\end{tabular}

Note-The abstractness classification scheme was adopted from Mosenthal (1996). 
Table 2

Frequency of the Question Items and Their Mean Item Difficulty (With Standard Deviations) As a Function of Passage Features (in Categorical Levels) for the 7th-9th and 10th-12th Grade Levels of the Gates-MacGinitie Reading Test

\begin{tabular}{|c|c|c|c|c|c|c|c|}
\hline \multirow[b]{2}{*}{ Passage Feature } & \multirow[b]{2}{*}{ Range } & \multicolumn{3}{|c|}{ 7th-9th } & \multicolumn{3}{|c|}{ 10th-12th } \\
\hline & & $n$ & $M$ & $S D$ & $n$ & $M$ & $S D$ \\
\hline \multirow[t]{5}{*}{ Number of propositions } & Less than 10.00 & 26 & .61 & .15 & 14 & .55 & .11 \\
\hline & $10.00-19.99$ & 49 & .67 & .16 & 32 & .61 & .15 \\
\hline & $20.00-29.99$ & 21 & .73 & .15 & 30 & .63 & .12 \\
\hline & $30.00-39.99$ & 0 & NA & & 16 & .66 & .09 \\
\hline & $40.00-50.00$ & 0 & NA & & 4 & .62 & .10 \\
\hline \multirow[t]{5}{*}{ Number of words/sentence } & Less than 10.00 & 8 & .71 & .15 & 0 & NA & \\
\hline & $10.00-19.99$ & 62 & .68 & .16 & 49 & .64 & .13 \\
\hline & $20.00-29.99$ & 13 & .75 & .12 & 31 & .59 & .13 \\
\hline & $30.00-39.99$ & 9 & .52 & .09 & 8 & .62 & .07 \\
\hline & $40.00-49.99$ & 4 & .55 & .04 & 8 & .53 & .07 \\
\hline \multirow[t]{5}{*}{ Log word frequency minimum } & Less than 0.40 & 20 & .60 & .14 & 20 & .56 & .09 \\
\hline & $0.40-0.79$ & 40 & .70 & .15 & 42 & .61 & .14 \\
\hline & $0.80-1.19$ & 28 & .65 & .16 & 24 & .65 & .13 \\
\hline & $1.20-1.59$ & 5 & .68 & .12 & 4 & .63 & .10 \\
\hline & $1.60-1.99$ & 3 & .88 & .03 & 6 & .69 & .13 \\
\hline \multirow[t]{4}{*}{ Flesch Reading Ease } & Less than 25.00 & 4 & .54 & .04 & 3 & .48 & .09 \\
\hline & $25.00-49.99$ & 5 & .60 & .12 & 26 & .54 & .10 \\
\hline & $50.00-74.99$ & 56 & .68 & .15 & 61 & .65 & .12 \\
\hline & $75.00-100$ & 31 & .67 & .16 & 6 & .69 & .13 \\
\hline
\end{tabular}

levels. The table shows that the 7th-9th and 10th-12th grade levels include a variety of passage types that differ in terms of reading ease, average sentence length, word frequency, and propositional density.

We also classified the passages used in the tests into three different text genres. Narrative passages tend to describe relatively mundane events with which most people have some familiarity from a personal perspective. Expository passages tend to describe historical, social, and/or scientific facts from a nonpersonal, objective perspective. The third category is poetry. According to our classification, the GMRT 7th-9th grade level contains 11 narrative passages, 15 expository passages, and 2 poems. The GMRT 10th-12th grade level contains 13 narratives and 9 expository passages. Thus, both the 7th-9th and the 10th-12th grade level tests include a variety of passages, although the distributions differ somewhat across the two tests. Overall, this preliminary analysis indicated that both grade level tests appear to examine reading skill by including a range of different question types and passage features that vary in terms of genre, vocabulary difficulty, sentence length, and overall passage length.

\section{Contributions of Item and Text Features to Item Difficulty}

To explore the effects of individual-question features and passage features on item difficulty, we used HLM, which is suitable for analyzing data within a nested data structure. For example, the degree to which several factors (e.g., socioeconomic status, gender, class size, or school district) at different levels (e.g., level of individual students, level of classroom, level of school, etc.) influence an outcome variable can be analyzed using HLM. As such, HLM is often used in educational research whose aim is to understand the contribution of multiple factors on students' performance that cannot be readily analyzed using ordinary multiple regression (Lee, 2004).
The advantage of HLM is not limited only to research with people nested in educational and/or social conditions. Text comprehension research benefits from HLM because text comprehension research often involves nested data structures. HLM provides a methodological advantage when it is necessary to analyze the contribution of multiple factors across multiple levels in nested data. For example, Richter (2006) demonstrated the advantage of HLM over other statistical techniques when analyzing reading time data for sentences nested in a passage (see Richter, 2006, for a detailed discussion of HLM in reading comprehension research). As such, HLM is suited for the present study because it requires the analysis of the contribution of item-level factors and passage-level factors simultaneously.

Although the item types used in the GMRT may take on a number of generic forms, the difficulty level of an item will depend not only on the item itself, but also on the passage on the basis of which the item is constructed. In other words, the items are nested within a particular passage. This type of data structure requires an analysis in which the influence of Level 1 (i.e., individual-item) features, the influence of Level 2 (passage-level) features, and the interaction between Level 1 and Level 2 features (i.e., the influence of passage features on the contribution of individual features) is separated out, thereby justifying the use of a two-level HLM.

As a preliminary justification of the use of two-level HLM, a multiple regression was performed to check whether the proportion of variance accounted for by the Level 2 (passage-level) variables was large enough by entering number of propositions, minimum word frequency, and sentence length as predictor variables. With respect to the GMRT 7th-9th grade level, the three passage-level variables accounted for approximately $15 \%$ of variance $\left(R^{2}=.148\right)$. With respect to the GMRT 
Level 1 Model

$$
\begin{aligned}
& Y=B 0+B 1 *(\text { question } / \text { passage relations })+B 2 *(\text { Abstractness })+ \\
& B 3 *(\text { Falsifiability })+R
\end{aligned}
$$

Level 2 Model

$$
\begin{aligned}
& B 0=G 00+G 01 *(\text { Number of propositions })+G 02 *(\text { Sentence Length })+ \\
& G 03 *(\text { Average Min. Word Freq })+U 0 \ldots \ldots(2) \\
& B 1=G 10+G 11 *(\text { Number of propositions })+G 12 *(\text { Sentence Length })+ \\
& G 13 *(\text { Average Min. Word Freq })+U 1 \ldots \ldots(3) \\
& B 2=G 20+G 21 *(\text { Number of propositions })+G 22 *(\text { Sentence Length })+ \\
& G 23 *(\text { Average Min. Word Freq })+U 2 \ldots . .(4) \\
& B 3=G 30+G 31 *(\text { Number of propositions })+G 32 *(\text { Sentence Length })+ \\
& G 33 *(\text { Average Min. Word Freq })+U 3 \ldots . . .(5)
\end{aligned}
$$

Figure 1. Equations representing the two-level hierarchical linear modeling computed in this study.

10th-12th grade level, the three passage-level variables accounted for approximately $10 \%$ of variance $\left(R^{2}=.100\right)$. Hence, the analyses indicated that a moderate proportion of variance of the dependent variable (i.e., item difficulty) was accounted for by Level 2 units, warranting the use of a two-level HLM.

The two-level HLM used in this study models the effect of three item-level predictor variables (i.e., abstractness of the question, degrees of transformation required, and falsifiability of the distractors), three passage-level predictor variables (i.e., number of propositions in the passage, average sentence length, and minimum word frequency level), and the interactions between the variables across the levels (i.e., $3 \times 3$ interactions) on the individual item's difficulty level. We used the HLM 6, provided by Scientific Software International (2007). Figure 1 depicts the specific model tested.

We analyzed the GMRT 7th-9th and 10th-12th grade levels separately. Within the model, we used raw scores to represent item-level predictor variables (i.e., question/ passage relations, abstractness, and falsifiability), because they are categorical variables. However, we used grand mean centered scores to represent passage-level predictor variables (number of propositions, sentence length, and minimum word frequency) in order to facilitate the interpretation of the results. When passage-level predictor variables are grand mean centered, zero values in passagelevel variables represent the average score for passage features in the sample. In this way, the intercepts (i.e., G10, G20, and G30) in Equations 3, 4, and 5 estimating the effect of individual-item features (i.e., $B 1, B 2$, and $B 3$ ) on item difficulty represent the main effect of the individualitem features when the passage features are at the average level. Table 3 presents average passage features (i.e., number of propositions, words per sentence, minimum word frequency, and reading ease) for all the passages used in the GMRT 7th-9th and 10th-12th grade levels, respectively. If we used a raw score to represent passage features, instead of the grand mean centered score, the intercept would become the main effect of the item feature when the score of a given passage feature is at absolute zero. However, this would not be meaningful, because there is no such passage with a feature (i.e., sentence length or number of propositions) at zero.

In this model, the main effects of individual-item features on item difficulty were represented by the parameters $G 10, G 20$, and G30, the main effects of passage features on item difficulty by $G 01, G 02$, and $G 03$, and the interactions between item features and passage features by $G 11$, $G 12, G 13, G 21, G 22, G 23, G 31, G 32$, and $G 33$, respectively (see Table 4). We adopted a liberal criterion $(a=.1)$ during the interpretation of the reliability of the effects of predictor variables for two reasons. First, this analysis was exploratory, and we were willing to take a higher risk with respect to Type I errors. Second, sample size was limited by the length of the specific test we were analyzing, which was relatively small for analysis using HLM. This forced us to adopt a liberal criterion to ensure that we did not overlook the potential effects that may emerge in this particular analysis with a limited number of items.

Table 3

Average Passage Features of the Passages Used in the 7th-9th and 10th-12th Grade Levels for the Gates-MacGinitie Reading Test

\begin{tabular}{lcc}
\multicolumn{1}{c}{ Feature } & 7th-9th & 10th-12th \\
\hline Number of propositions & 13.29 & 19.14 \\
Number of words per sentence & 18.70 & 23.49 \\
Logarithm word frequency & & \\
$\quad$ (minimum per sentence) & 0.72 & 0.66 \\
Flesch Reading Ease & 68.47 & 55.57 \\
\hline
\end{tabular}


Table 4

Estimation of Fixed Effects Based on Hierarchical Linear Modeling (HLM) Analysis of the 7th-9th Grade Level of the Gates-MacGinitie Reading Test

\begin{tabular}{lcrrrr}
\hline \multicolumn{1}{c}{ Type of Fixed Effect } & HLM & Coefficient & $S E$ & $t$ Ratio & $p$ Value \\
\hline Intercept & $G 00$ & .667 & .031 & 21.24 & $.01^{* *}$ \\
Number of propositions & $G 01$ & .008 & .004 & 2.04 & $.05^{*}$ \\
Sentence length & $G 02$ & .008 & .006 & 1.42 & .17 \\
Word frequency minimum & $G 03$ & .311 & .156 & 1.99 & $.06^{*}$ \\
Passage/question (PQ) relation intercept & $G 10$ & -.027 & .016 & -1.66 & $.09^{*}$ \\
$\quad$ PQ relation $\times$ number of propositions & $G 11$ & .001 & .003 & 0.44 & .67 \\
PQ relation $\times$ sentence length & $G 12$ & .002 & .002 & 1.45 & .16 \\
PQ relation $\times$ word frequency & $G 13$ & -.033 & .046 & 0.72 & .48 \\
Abstractness intercept & $G 20$ & .018 & .012 & 1.50 & .14 \\
$\quad$ Abstractness $\times$ number of propositions & $G 21$ & -.001 & .002 & -0.40 & .69 \\
Abstractness $\times$ sentence length & $G 22$ & -.002 & .002 & -0.88 & .39 \\
Abstractness $\times$ word frequency & $G 23$ & .006 & .054 & 0.11 & .92 \\
Falsifiability intercept & $G 30$ & -.035 & .020 & -1.76 & $.09^{*}$ \\
$\quad$ Falsifiability $\times$ number of propositions & $G 31$ & -.038 & .003 & -1.10 & .28 \\
Falsifiability $\times$ sentence length & $G 32$ & -.012 & .003 & -3.46 & $.002^{* *}$ \\
Falsifiability $\times$ word frequency & $G 33$ & -.260 & .064 & -4.03 & $.001^{* *}$ \\
\hline${ }^{*} p<.1 .{ }^{* *} p<.05$. & & & & &
\end{tabular}

GMRT 7th-9th grade level. Table 4 indicates the fixed effects of three item-level and three passage-level predictor variables on item difficulty. First, the effect of the intercept $(G 00)$, although statistically significant, is not interesting, because this merely indicates the mean item difficulty score across all items. Second, with respect to the passage-related predictor variables, there were main effects of the number of propositions $(G 01)$ and the average minimum word frequency per sentence (G03). These findings indicate that questions based on passages with higher propositional density or with higher word frequency tend to be easier. This finding, which indicates that questions based on passages with a smaller number of propositions are more difficult, can be observed in Table 2, wherein the mean item difficulty score (i.e., the proportion correct) for the questions based on passages with fewer than 10 propositions is .61, and the mean item difficulty score for questions based on passages with more than 20 propositions is .73. This finding contradicts the results in Embretson and Wetzel (1987), who found an increase in difficulty level with the increase in the number of propositions. One possible explanation for this somewhat anomalous finding is that it emerges from the intercorrelations between passage features (i.e., sentence length and word frequency). We will discuss the possible influence of intercorrelations in a subsequent section.

With respect to item-level variables (Level 1), no variables contributed to item difficulty apart from a marginal contribution of passage/question relation (G10) and falsifiability (G30). The contribution of the passage/question relation was negative, indicating that items that contain a more indirect relation between source passage and question content (i.e., inference or bridging inference, as opposed to text-based items) tend to be more difficult. Interestingly, the contribution of falsifiability was also negative, indicating that performance tends to be lower when at least one distractor is falsifiable. However, the negative effect of falsifiability interacted with minimum word frequency (G32) and sentence length (G33), so that the negative effect of falsifiability was magnified when minimum word frequency was higher and/or sentence length was greater than average. We will present more detailed discussion on these interactions later.

Table 5 presents the estimation of the error components in the analysis. The results indicate that the error term associated with the equation estimating the slope for the passage/question relation is significantly different from zero. This result suggests that there is significant random variability in the relationship between item difficulty and passage/question relations across different passages. On the other hand, the random effect of passage on item difficulty, the relation between item difficulty and the abstractness of the question, and the relation between item difficulty and falsifiability were not significant.

In summation, the analyses indicate that the difficulty level of the items in the 7th-9th grade level GMRT is determined primarily by passage features, as opposed to features of individual questions. The specific passage features that appear to have significant impact on item difficulty are the number of propositions in a passage

Table 5

Estimation of Random Effects (Error Components) on the 7th-9th Grade Level of the Gates-MacGinitie Reading Test

\begin{tabular}{lccccc}
\hline \multicolumn{1}{c}{ Type of Random Effects } & HLM & $S D$ & $d f$ & $\chi^{2}$ & $p$ Value \\
\hline Intercept 1 & $U 0$ & .054 & 6 & 8.08 & .23 \\
Slope for passage/question relation & $U 1$ & .038 & 6 & 13.33 & $.04^{* *}$ \\
Slope for abstractness & $U 2$ & .003 & 6 & 8.11 & .23 \\
Slope for falsifiability & $U 3$ & .039 & 6 & 5.71 & $>.50$ \\
${ }^{* *} p<.05$. & & & & &
\end{tabular}


Table 6

Correlations Between Passage Features

\begin{tabular}{|c|c|c|c|c|c|c|}
\hline & \multicolumn{2}{|c|}{$\begin{array}{l}\text { Number of } \\
\text { Propositions }\end{array}$} & \multicolumn{2}{|c|}{$\begin{array}{c}\text { Number of Words } \\
\text { per Sentence }\end{array}$} & \multicolumn{2}{|c|}{$\begin{array}{l}\text { Minimum } \\
\text { Word } \\
\text { Frequency }\end{array}$} \\
\hline & $\begin{array}{c}\text { Level } \\
7 / 9\end{array}$ & $\begin{array}{l}\text { Level } \\
10 / 12\end{array}$ & $\begin{array}{l}\text { Level } \\
7 / 9\end{array}$ & $\begin{array}{l}\text { Level } \\
10 / 12\end{array}$ & $\begin{array}{c}\text { Level } \\
7 / 9\end{array}$ & $\begin{array}{l}\text { Level } \\
10 / 12\end{array}$ \\
\hline Number of words per sentence & $-.332^{* * *}$ & $-.335^{\dagger}$ & & & & \\
\hline Minimum word frequency & $.200 \ddagger$ & $.409^{\dagger}$ & $-.754^{\dagger}$ & $-.504 \dagger$ & & \\
\hline Flesch Reading Ease & $.238^{* *}$ & $.480^{\dagger}$ & $-.791^{\dagger}$ & $-.516^{\dagger}$ & $.738^{\dagger}$ & $.664^{\dagger}$ \\
\hline
\end{tabular}

${ }^{* *} p<.05 . \quad{ }^{* * *} p<.01 . \quad{ }^{\dagger} p<.001 . \quad \ddagger$ n.s.

and the level of minimum word frequency. The positive influence of propositional density on item difficulty is unexpected. One potential explanation comes from the intercorrelations among passage features. Table 6 presents correlations among the four passage features used in this analysis. These intercorrelations between passage features are also somewhat unexpected. For example, the number of propositions in the passage is negatively correlated with the number of words per sentence and is positively correlated with reading ease. These correlations indicate that passages with more propositions contain shorter sentences and are easier to read. Thus, the positive influence of propositional density on item difficulty might be due to irregular features of a highly specific set of passages used in the GMRT 7th-9th grade level test.

With respect to the influence of minimum word frequency, items associated with a passage containing lowfrequency words tend to be more difficult. The coefficient for minimum word frequency (.31; see Table 4$)$ and the variability of minimum word frequency (minimum, 0 ; maximum, 1.80) together indicate that item difficulty can vary more than .5 points in the score of item difficulty $(.31 \times$ $1.80=.54$ ) as a function of word frequency alone.

In order to follow up this finding, we performed a oneway ANOVA on item difficulty as a function of the minimum word frequency, using the median split technique. The median value of minimum word frequency was 0.70 . There were 46 items based on passages whose minimum word frequency was below the median, and there were 50 items based on passages whose minimum word frequency was above median. The ANOVA indicated a significant difference in item difficulty as a function of word frequency $\left[F(1,94)=4.01, M S_{\mathrm{e}}=.023, p<.05\right]$, indicating that questions based on passages with high-frequency words $(M=.70, S D=.15)$ are easier than questions based on passages with low-frequency words $(M=.64, S D=$ .15). Hence, the HLM and ANOVA converge to support the conclusion that word frequency in the passage has a strong effect on item difficulty.

In addition to passage features, item difficulty appears to be influenced by individual-item features, albeit at a marginal level. First, the relations between question and source passage appear to moderate item difficulty, so that items that require more integration or knowledge-based inferences appear to be more difficult. Given that this effect does not interact with passage-level variables, the trend that more integrative or inferential questions tend to be more difficult is assumed to be fairly stable, even though the effect is relatively small. This finding is in line with Embretson and Wetzel (1987), who found an effect of question/passage transformation, using Anderson's (1972) scheme.

Falsifiability of the distractors also influences item difficulty, although the effect is marginal. A negative coefficient $(-.035)$ of falsifiability indicates that an item tends to be more difficult when one or more distractors are falsifiable than when no distractors are falsifiable, a rather surprising trend. However, this effect interacts negatively with two passage features (i.e., sentence length and word frequency). This interaction means that under some circumstances, the negative effect of falsifiability is alleviated. Specifically, the trend that the presence of falsifiable distractors makes the item more difficult becomes small when sentence length is shorter or word frequency is lower than average. Figure 2 illustrates the nature of the interactions between distractor falsifiability and word frequency and between distractor falsifiability and sentence length. The figures plot the effect of distractor falsifiability separately for high-frequency word passages (average across 50 items based on high word frequency passages) and low word frequency passages (average across 46 items based on low word frequency passages; top panel) and for short sentence passages (average across 48 items based on short sentence passages) and long sentence passages (average across 48 items based on long sentence passages; bottom panel) using a median split based on word frequency or sentence length. These figures show that the effect of distractor falsifiability on item difficulty changes as a function of sentence length or word frequency of a passage. However, it should be noted that the effect of falsifiability itself is marginal and its interactions with passage-level variables seem to make the influence of falsifiable distractors somewhat trivial.

GMRT 10th-12th grade level. Table 7 presents the fixed effect of three item-level and three passage-level predictor variables on item difficulty. There were no significant main effects of the passage features. In addition, there were no significant main effects of the individual items. Only the abstractness of the question (G20) showed small interactive effects with the number of propositions (G21), sentence length (G22), and minimum word frequency (G23).

More specifically, the abstractness of the question influences item difficulty in different ways depending on propositional density, word frequency, and sentence length of the passage. In order to pursue the nature of these in- 
Item Difficulty As a Function of the Falsifiability of a Question and Word Frequency of a Passage

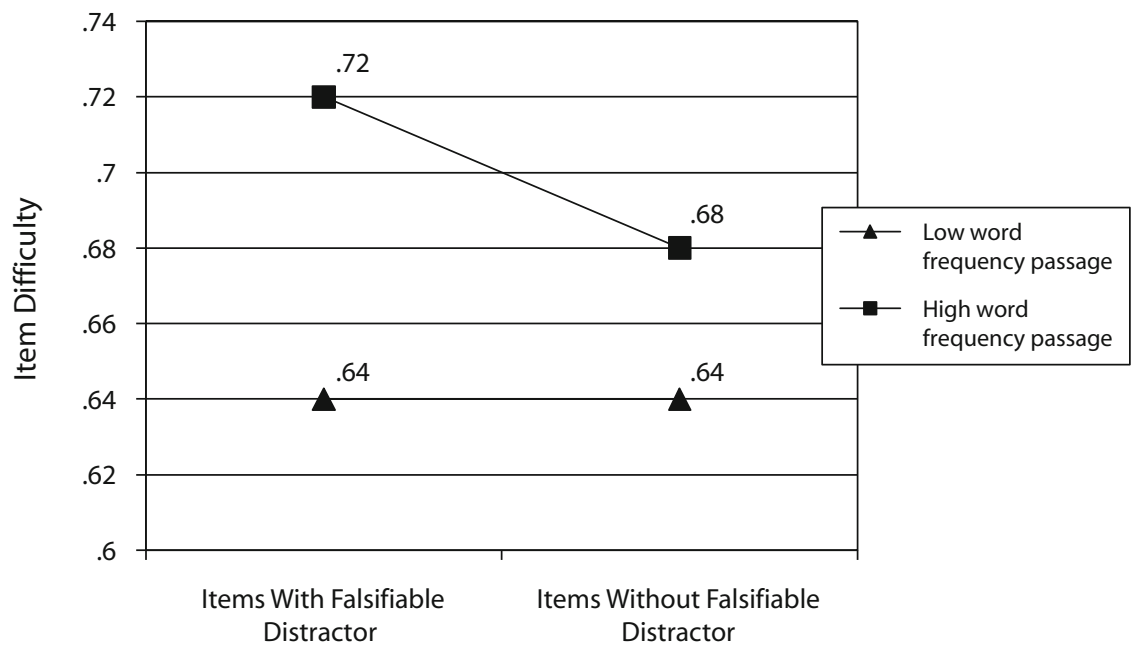

Item Difficulty As a Function of the Falsifiability of a Question and Sentence Length of a Passage

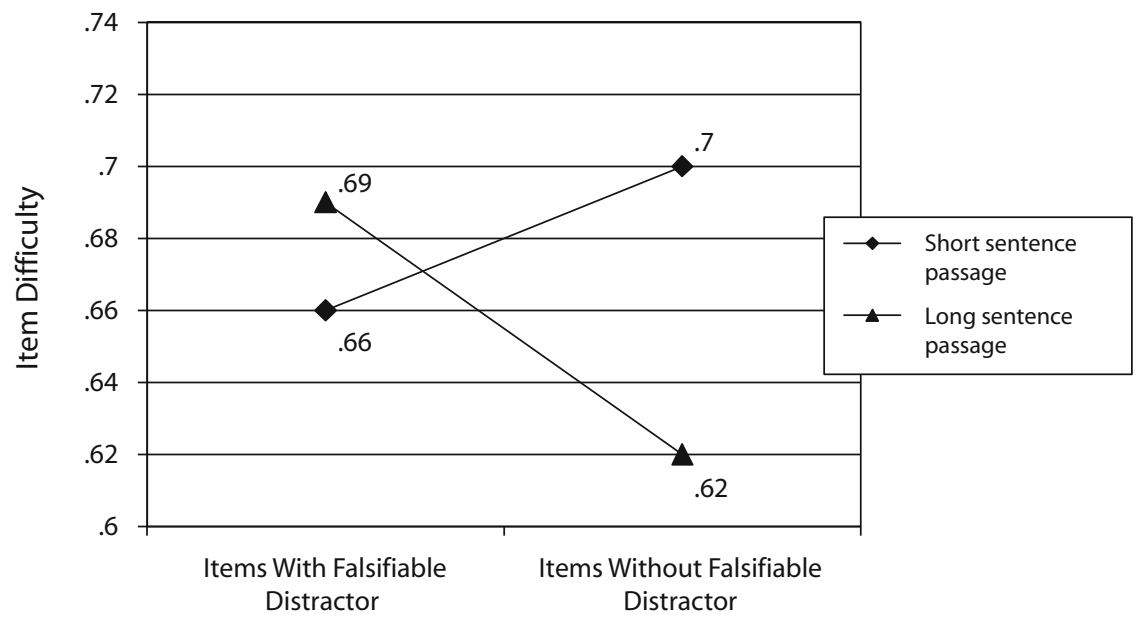

Figure 2. Interactions between distractor falsifiability and passage features on item difficulty for the 7th-9th grade level of the Gates-MacGinitie Reading Test.

Table 7

Estimation of Fixed Effects Based on Hierarchical Linear Modeling (HLM) Analysis of the 10th-12th Grade Level of the Gates-MacGinitie Reading Test

\begin{tabular}{|c|c|c|c|c|c|}
\hline Type of Fixed Effect & HLM & Coefficient & $S E$ & $t$ Ratio & $p$ Value \\
\hline Intercept & $G 00$ & .655 & .049 & 13.14 & $.00^{* *}$ \\
\hline Number of propositions & $G 01$ & .006 & .005 & 1.27 & .22 \\
\hline Sentence length & $G 02$ & -.003 & .005 & -0.53 & .60 \\
\hline Word frequency minimum & $G 03$ & .097 & .138 & 0.71 & .49 \\
\hline Passage/question (PQ) relation intercept & $G 10$ & -.008 & .014 & -0.60 & .56 \\
\hline PQ relation $\times$ number of propositions & $G 11$ & -.000 & .001 & -0.13 & .89 \\
\hline PQ relation $\times$ sentence length & $G 12$ & -.001 & .001 & -1.32 & .20 \\
\hline PQ relation $\times$ word frequency & $G 13$ & -.062 & .043 & -1.45 & .17 \\
\hline Abstractness intercept & $G 20$ & -.008 & .005 & -1.41 & .18 \\
\hline Abstractness $\times$ number of propositions & $G 21$ & -.001 & .0005 & -2.14 & $.04^{* *}$ \\
\hline Abstractness $\times$ sentence length & $G 22$ & .002 & .0005 & 4.12 & $.00^{* *}$ \\
\hline Abstractness $\times$ word frequency & $G 23$ & .048 & .008 & 6.22 & $.00^{* *}$ \\
\hline Falsifiability intercept & $G 30$ & .002 & .017 & 0.12 & .91 \\
\hline Falsifiability $\times$ number of propositions & $G 31$ & -.002 & .002 & -1.02 & .32 \\
\hline Falsifiability $\times$ sentence length & $G 32$ & -.001 & .001 & -0.45 & .66 \\
\hline Falsifiability $\times$ word frequency & $G 33$ & .051 & .080 & 0.63 & .53 \\
\hline
\end{tabular}

${ }^{* *} p<.05$. 
Item Difficulty As a Function of the Abstractness of a

Question and the Propositional Density of a Passage

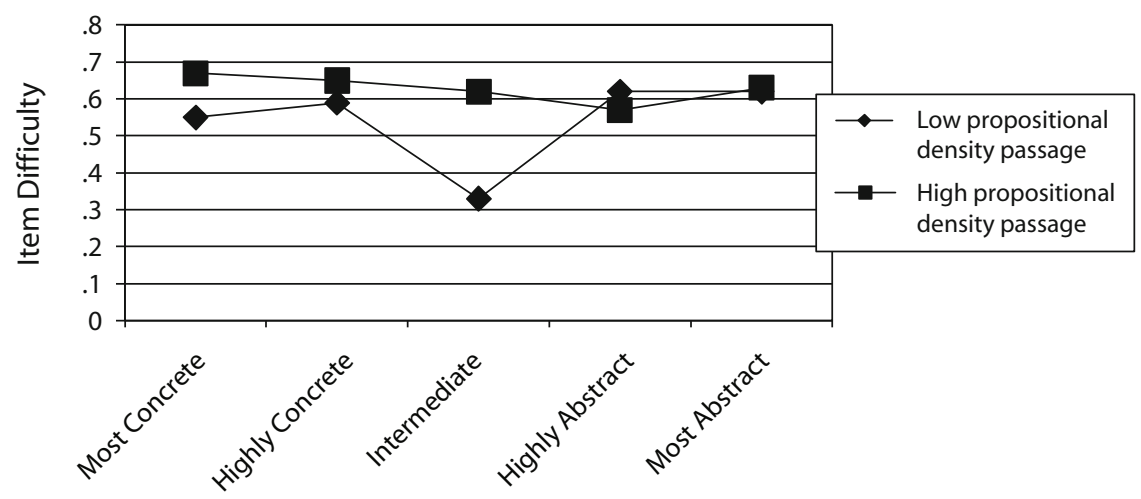

Item Difficulty As a Function of the Abstractness of a Question and Sentence Length of a Passage

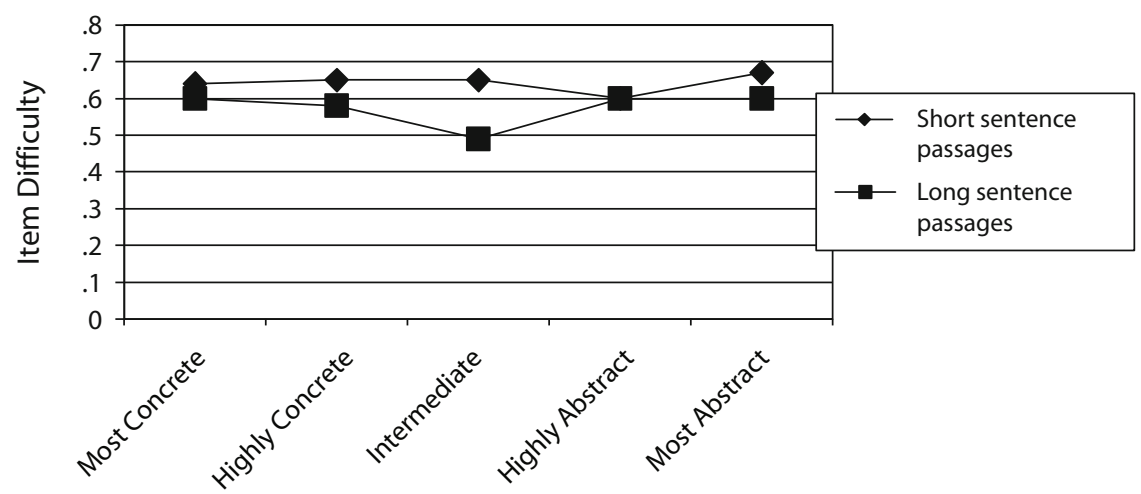

Item Difficulty As a Function of the Abstractness of a Question and Word Frequency of a Passage

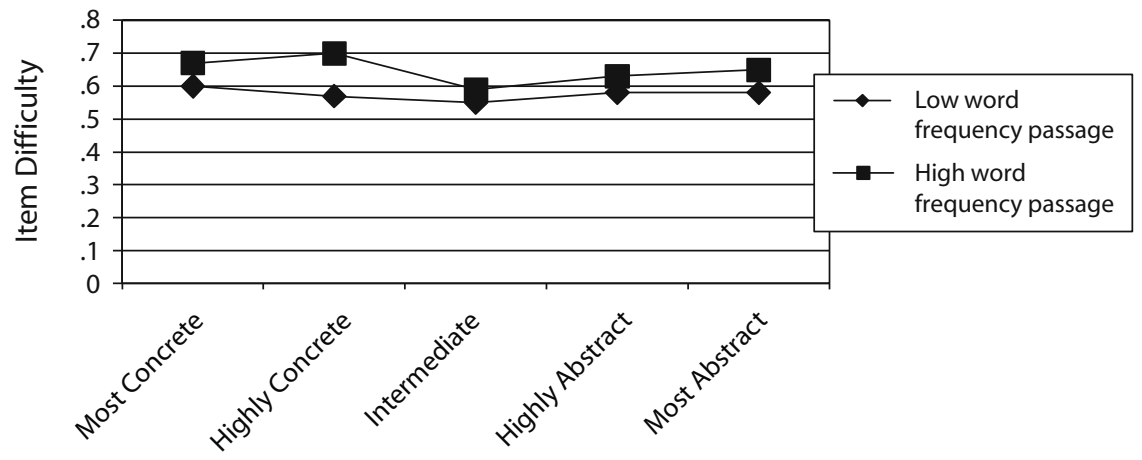

Figure 3. Interactions between question abstractness and passage features on item difficulty for the 10th-12th grade level of the Gates-MacGinitie Reading Test.

teractions, in Figure 3, we plotted the item difficulty for questions with different levels of abstractness separately for high or low propositional density passages (top panel), short or long sentence passages (middle panel), and low or high word frequency passages (bottom panel), using median splits.

As is shown in Figure 3, the influence of abstractness of the question, if any, is mostly due to the intermediate questions; questions with intermediate abstractness tend to be more difficult than other types of questions (more abstract or more concrete questions). Furthermore, this effect appears to change depending on the features of the source passage (e.g., propositions, sentence length, and word frequency). Therefore, the effect of question abstractness may be specific to a few particular questions. In any event, it is difficult to conclude whether abstractness of questions has any systematic influence on item difficulty of the 10th-12th grade level of the GMRT. 
Table 8

Estimation of Random Effects (Error Components) on the 10th-12th Grade Level of the Gates-MacGinitie Reading Test

\begin{tabular}{lccccc}
\hline \multicolumn{1}{c}{ Type of Random Effects } & HLM & $S D$ & $d f$ & $\chi^{2}$ & $p$ Value \\
\hline Intercept 1 & $U 0$ & .167 & 8 & 29.06 & $.001^{* *}$ \\
Slope for passage/question relation & $U 1$ & .037 & 8 & 26.55 & $.001^{* *}$ \\
Slope for abstractness & $U 2$ & .021 & 6 & 12.98 & .112 \\
Slope for falsifiability & $U 3$ & .026 & 6 & 15.00 & $.059^{*}$ \\
\hline${ }^{*} p<.1 . \quad{ }^{* *} p<.05$. & & & & &
\end{tabular}

Table 8 presents the results of the random effects estimation. The error terms associated with the intercept $(U 0)$, the slope of transformation (U1), and falsifiability (U3) were all significantly different from zero. Thus, overall, there are significant random fluctuations in item difficulty, the relation between item difficulty and passage/question relation, and the relation between item difficulty and falsifiability across different passages. Only the relation between item difficulty and the abstractness of the question appears to have a relatively small random fluctuation across passages. In summary, the HLM analyses on the 10th-12th grade level of the GMRT indicate virtually no presence of systematic influences of passage and/or item features on item difficulty.

\section{Comparison Between the 7th-9th and the 10th-12th Grade Levels of the GMRT}

In this section, we will compare the 7th-9th and the 10th-12th grade levels of the GMRT in terms of the results of an HLM analysis, quantitative comparisons, and qualitative observations of the materials used in the test. The HLM analysis on item difficulty of the GMRT 7th-9th grade level indicates that the difficulty level of items in the GMRT 7th-9th grade level is primarily influenced by passage features, as opposed to individual-question features. In particular, word frequency has a large impact on the level of item difficulty, as indicated by the main effect and its interaction with the falsifiability of distractors, an item-level variable.

In contrast to the GMRT 7th-9th grade level, the effects of individual-item features and passage features on item difficulty are less systematic for the GMRT 10th-12th grade level. That is, only the interactions between passage features and the abstractness of the questions has significant influences on item difficulty. However, the interaction between the abstractness of the questions and passage features may be due to a few specific comprehension questions, as is indicated by the follow-up analysis. Furthermore, all the fixed effects were very small, and there were significant random fluctuations across all the predictor variables. Hence, the item difficulty levels of the GMRT 10th-12th grade level are less systematically predicted by item and passage features used in this study, as compared with the GMRT 7th-9th grade level. This difference may be partly related to the amount of variability within the two target populations (i.e., 7th-9th graders and 10th-12th graders). However, the difference may, at least in part, be due to the individual items and passages. To explore the issue of whether these two levels of the test involve different test features, we directly compared item and passage features of the two tests.

With respect to the individual item differences, chi square analyses were conducted to examine whether the frequency of the different types of questions differs across the tests targeting 7th-9th and 10th-12th grades (see Table 1). The analyses indicated that the distribution of different types of questions differs between the two tests according to passage/question relations $\left[\chi^{2}(3)=\right.$ $19.37, p<.001]$ and the number of falsifiable distractors $\left[\chi^{2}(3)=9.96, p<.05\right]$. These two tests appear not to differ with regard to abstractness $\left[\chi^{2}(4)=9.48, p>.05\right]$. Thus, there are a larger number of questions that require a higher level of processing (i.e., bridging inference and knowledge-based inference questions) in the test targeting 10th-12th graders. Also, the test for 10th-12th grade students appears to contain a larger number of questions in which no distractor is falsifiable.

We also conducted chi square analyses to examine whether there is a difference in the frequency distribution of different passage types between the two tests (see Table 2). The analyses indicate that the distribution of different types of passages differs between the two tests according to propositional density $\left[\chi^{2}(4)=28.76, p<\right.$ $.001]$, sentence length $\left[\chi^{2}(4)=18.28, p<.001\right]$, and reading ease $\left[\chi^{2}(3)=31.047, p<.001\right]$. These two tests appear not to differ with regard to minimum word frequency $\left[\chi^{2}(4)=1.47, p>.1\right]$. The comparison indicates that the 10th-12th grade level test appears to use structurally more complex passages, as is indicated by a greater number of propositions in the text, the longer sentences in the passages, and a lower reading ease score. Surprisingly, the two tests did not differ in terms of minimum word frequency. More interesting, and perhaps of importance, is that the 10th-12th grade level test has a more restricted range of sentence length and contains longer sentences than does the 7th-9th grade level test. The range restriction in passage difficulty used in the 10th-12th grade level test is also represented in the reading ease measure, which is computed on the basis of sentence length and word length, a proxy measure for word frequency. Whereas the reading ease level varies from 10.53 to 100 in the 7 th-9th grade level test, it varies from 14.88 to 79.18 in the 10th-12th grade level test.

This comparison, together with the results of HLM, leads us to conclude that the GMRT 7th-9th grade level differentiates students' reading ability in terms of the difficulty of the passage that a reader can comprehend, as opposed to how deeply a reader can comprehend the 
passage content. Our observations of individual passages support this notion. For example, in the 7th-9th grade level test (MacGinitie \& MacGinitie, 1989), Passage 13 in Form $\mathrm{K}$ describes the highly technical issue of how large industrial British towns in the early 19th century facilitated the spreading of cholera. The passage comprises 91 words broken down into only two sentences. One sentence is more than 60 words long and is composed of several technical words. There are four questions (i.e., Questions 44-47) based on this passage, and their average item difficulty is .54 , roughly one standard deviation lower than mean item difficulty of all the items in this test.

In contrast, Passage 5 in Form $\mathrm{K}$ describes a very common event involving a young child's curiosity about a veterinarian's stethoscope. The passage uses generally shorter sentences. The longest sentence in this example is 21 words. There are five questions (i.e., Questions 14-18) based on this passage and their average item difficulty is .83 , more than one standard deviation above the mean. These examples indicate that one way, possibly the primary way, that variability in item difficulty is manipulated in the test is through the inclusion of extremely difficult passages.

The observation that passage difficulty is increased by using a passage composed of longer sentences and lowfrequency words at the same time (see the first example) might explain the failure to find a negative influence of sentence length on item difficulty in the HLM analysis on the 7th-9th grade level test. Intercorrelations among passage features in Table 6 show a strong negative correlation between word frequency and sentence length $(r=$ $-.754)$. Hence, the negative influence of sentence length on item difficulty may be overshadowed by a large positive influence of word frequency, because they are strongly negatively correlated. Thus, both the quantitative analysis and qualitative observation converge to indicate that item difficulty in the 7th-9th grade level test is likely to be manipulated primarily by word difficulty and sentence complexity. These processes are relatively shallow when viewed in the context of more extended text comprehension as a whole.

The types or aspects of reading comprehension ability measurement emphasized in the GMRT 10th-12th grade level are less clear, because no passage or item factors have significant influences on item difficulty. Nonetheless, it is relatively clear that the GMRT 10th-12th grade level does not have as strong an emphasis on the measurement of "how difficult a passage a reader can comprehend" as does the GMRT 7th-9th grade level, because variability in passage difficulty is much smaller. One possibility is that the GMRT 10th-12th grade level has more emphasis, or at least aimed at having an emphasis, on the measurement of a deeper comprehension of generally more difficult passages. This possibility is partially supported by the larger number of more inference-based questions in the test. But as far as our analyses are concerned, the individual-question types seem not to have much systematic influence on item difficulty.

\section{CONCLUSION}

Overall, detailed item analyses based on cognitive processing models provide useful information about the nature of standardized reading comprehension tests such as the GMRT. In particular, the analyses provide some insights into how the test measures the reading comprehension ability of a wide variety of readers by varying item features to produce items with different levels of difficulty. At a more specific level, the analyses reveal several interesting observations about both levels of the GMRT. First, the difficulty level of tests that target two different grade levels seems to be manipulated with both passage features and the type of questions. A larger number of inferential questions are found in the test targeting higher grade level students. In addition, more difficult passages are used in the test targeting higher grade level students.

Another interesting finding concerns the effects of item and passage features on item difficulty within the tests. The results of the HLM analyses indicate that the item difficulty for the test targeting lower grade level students is influenced largely by passage features, instead of the type of questions. Although there are some effects of question type (i.e., passage/question relation and falsifiability) on item difficulty, the effects are relatively small and often interact with passage features.

A final interesting point to be noted is the influence of falsifiability of distractor options on item difficulty, at least for the 7th-9th grade level test. This finding is consistent with Embretson and Wetzel's (1987) findings and indicates that the quality of distractor options in multiple-choice tests is an important attribute of an assessment. Unfortunately, this result implies that the GMRT 7th-9th grade level is influenced by a variable that has little to do with reading comprehension, given that eliminating improbable answer options is associated more strongly with general test-taking skills than with reading comprehension skills.

These findings by no means invalidate the GMRT. The tests contain a variety of passages with varying ranges of difficulty, differing in a number of dimensions. Also, the tests contain a number of different types of questions, most of which cannot be answered by simply eliminating distractors. Thus, these results indicate that the test extensively measures many different subcomponents underlying reading comprehension in the context of a variety of reading circumstances.

Perhaps one reason that the test fails to be theoretically focused and capable of tapping into specific processes emerges from its need to assess the reading ability levels of students with a variety of ability levels, ranging from students with fairly poor skills to students having quite advanced skills. The test, by its very nature, cannot be focused on specific processing components, because of the large variability in the target population to which the test needs to be sensitive. For a test to effectively evaluate broad reading ability of a diverse population, it must contain a large number of items that are differently sensitive to variability in different types of reading comprehension processes and ability present in the target population. 
The findings presented in this article generate three take-home messages. The first message is mainly for test makers. It is clear that both tests include a range of test materials in terms of the various item and passage features. On the one hand, the numerous combinations of the item features observed in this analysis may represent the test makers' effort to measure students' reading ability in an exhaustive manner. On the other hand, some specific groups of extreme items included in the test can also be perceived as the test makers' strategy to increase the range of test discrimination by including almost unnaturally easy or difficult items. These examples include items with several falsifiable distractors (to make an item easy) and items based on passages composed of extremely long sentences with low-frequency words. To the extent that reading comprehension ability represents complex combinations of interrelated elementary cognitive processes (e.g., decoding, vocabulary, reasoning skill, etc.), the inclusion of these item types with extreme characteristics may not undermine the overall integrity of the test when represented using psychometric parameters. Yet this type of test construction strategy is likely to produce test scores that are difficult to interpret in any theoretically and educationally meaningful ways, a point raised by several researchers (e.g., Daneman \& Hannon, 2001; Magliano et al., 2007; VanderVeen et al., 2007). In this sense, test makers should put forth an effort to maintain overall discrimination and test sensitivity without compromising the theoretical validity of the assessment. Such an attempt would require active incorporation of theories and findings on text comprehension into test construction and adopting newly emerging techniques of test construction, such as adoptive testing (Weiss \& Kingsbury, 1984) and/or application of evidencecentered design (Mislevy, Steinberg, Breyer, Almond, \& Johnson, 1999).

The second message is for the consumer of standardized reading ability tests such as the GMRT. The consumer should be aware of the specific strengths and limitations of this test. More specifically, the GMRT appears to be suited for assessing a broad range of abilities involved in reading comprehension from a variety of text materials in a broad stroke. The GMRT is not suited for the diagnostic purpose of identifying specific comprehension problems in students (cf. Magliano et al., 2007). It is difficult to infer from the test scores alone what would be the source of the comprehension problems of specific students with a particular score level.

The final message is for researchers on text comprehension and educational assessment. This article indicates that a detailed item analysis of standardized tests based on the cognitive processing model provides some useful information about specific features of the test, hence informing researchers, educators, and test makers about issues for educational assessment. Our hope is that this article will help to stimulate research involving item analysis with a variety of sophisticated techniques that will inform users about the strengths and weaknesses of various assessment tools used to evaluate students.

\section{AUTHOR NOTE}

This research was supported by the Institute for Education Sciences (IES R3056020018-02). Correspondence concerning this article should be addressed to Y. Ozuru, Learning Sciences Research Institute, 1007 W. Harrison Street, Room 2048 (MC 057), Chicago, IL 60607-7137 (e-mail: yasozuru@uic.edu or y.ozuru@mail.psyc.memphis.edu).

\section{REFERENCES}

Adams, M., \& Collins, A. (1979). A schema-theoretic view of reading. In R. O. Freedle (Ed.), New directions in discourse processing (pp. 1-22). Norwood, NJ: Ablex.

ANDERSON, R. C. (1972). How to construct achievement tests to assess comprehension. Review of Educational Research, 42, 145-170.

BridgEman, B., \& Rock, D. A. (1993). Relationships among multiplechoice and open-ended analytical questions. Journal of Educational Measurement, 30, 313-329.

CAMPBell, J. R. (1999). Cognitive processes elicited by multiple-choice and constructed-response questions on an assessment of reading comprehension. Unpublished doctoral dissertation, Temple University, Philadelphia.

Charniak, E. (2000). A maximum-entropy-inspired parser. In Proceedings of the First Annual Meeting of the North American Chapter of the Association for Computational Linguistics (pp. 132-139). San Francisco: Morgan Kaufman.

Cordon, L. A., \& DAY, J. D. (1996). Strategy use on standardized reading comprehension tests. Journal of Educational Psychology, 88, 288-295.

Daneman, M., \& Hannon, B. (2001). Using working memory theory to investigate the construct validity of multiple-choice reading comprehension tests such as the SAT. Journal of Experimental Psychology: General, 130, 208-223.

EMBRETSON, S. E. (1998). A cognitive design system approach to generating valid tests: Application to abstract reasoning. Psychological Methods, 3, 300-396.

Embretson, S. E., \& ReISE, S. P. (2000). Item response theory for psychologists. Mahwah, NJ: Erlbaum.

Embretson, S. E., \& Wetzel, C. D. (1987). Component latent trait models for paragraph comprehension. Applied Psychological Measurement, 11, 175-193.

ERICsSON, K. A., \& SimON, H. A. (1993). Protocol analysis: Verbal reports as data. Cambridge, MA: MIT Press.

Fletcher, M. J. (2006). Measuring reading comprehension. Scientific Study of Reading, 10, 323-330.

Gates-MacGinitie Reading Tests: Technical reportfor Gates-MacGinitie Reading Tests Forms K and L (1989). Chicago: Riverside.

Gorin, J. S., Embretson, S. E., \& Sheehan, K. (2002, April). Cognitive and psychometric modeling of text-based reading comprehension GRE-V items. Paper presented at the 2002 Annual Meeting of the National Council on Measurement in Education, New Orleans.

Graesser, A. C., McNamara, D. S., Louwerse, M. M., \& Cai, Z. (2004). Coh-Metrix: Analysis of text on cohesion and language. Behavior Research Methods, Instruments, \& Computers, 36, 193-202.

Hannon, B., \& Daneman, M. (2001). A new tool for measuring and understanding individual differences in the component processes of reading comprehension. Journal of Educational Psychology, 93, 103-128.

Just, M. A., \& CARPEnTER, P. A. (1992). A capacity theory of comprehension: Individual differences in working memory. Psychological Review, 99, 122-149.

Katz, S., Blackburn, A. B., \& Lautenschlager, G. J. (1991). Answering reading comprehension items without passages on the SAT when items are quasi-randomized. Educational \& Psychological Measurement, 51, 747-754.

Kintsch, W. (1988). The use of knowledge in discourse processing: A construction integration model. Psychological Review, 95, 163-182.

KInTSCH, W. (1998). Comprehension: A paradigm for cognition. Cambridge: Cambridge University Press.

KinTSCH, W., \& VAN DiJK, A. (1978). Toward a model of text comprehension and production. Psychological Review, 85, 363-394.

LEE, J. (2004). Evaluating the effectiveness of instructional resource 
allocation and use: IRT and HLM analysis of NAEP teacher survey and student assessment data. Studies in Educational Evaluation, 30, 175-199.

MacGinitie, W. H., \& MacGinitie, R. K. (1989). Gates-MacGinitie Reading Test (3rd ed.). Itasca, IL: Riverside.

Magliano, J. P., Millis, K. K., Ozuru, Y., \& McNamara, D. S. (2007). A multidimensional framework to evaluate reading assessment tools. In D. S. McNamara (Ed.), Reading comprehension strategies: Theory, interventions, and technologies (pp. 107-136). Mahwah, NJ: Erlbaum.

ManharT, J. J. (1996, April). Factor analytic method for determining whether multiple-choice and constructed response tests measure the same construct. Paper presented at the 1996 Annual Meeting of the National Council on Measurement in Education, New York.

MARSLEN-WILSON, W. (1975). Sentence perception as an interactive parallel process. Science, $\mathbf{1 8 9}, 226-228$.

Messick, S. (1989). Validity. In R. L. Linn (Ed.), Educational measurement (3rd ed., pp. 13-103). New York: Macmillan.

Messick, S. (1996a). Standards-based score interpretation: Establishing valid grounds for valid inferences. In L. Crocker \& M. Zieky (Eds.), Proceedings of the Joint Conference on Standard Setting for Large Scale Assessments (Vol. 2, pp. 291-305). Washington, DC: U.S. Government Printing Office.

Messick, S. (1996b). Validity of performance assessment. In G. Philips (Ed.), Technical issues in large-scale performance assessment (pp. 1-18). Washington, DC: National Center for Education Statistics.

Mislevy, R. J. (1994). Evidence and inference in educational assessment. Psychometrika, 59, 439-483.

Mislevy, R. J. (1995). Probability-based inference in cognitive diagnosis. In P. D. Nichols, S. F. Chipman, \& R. L. Brennan (Eds.), Cognitively diagnostic assessment (pp. 43-71). Hillsdale, NJ: Erlbaum.

Mislevy, R. J., Steinberg, L. S., Breyer, F. J., Almond, R. G., \& Johnson, L. (1999, September). Making sense of data from complex assessments. Paper presented at the 1999 CRESST Conference, Los Angeles.

Mosenthal, P. (1996). Understanding the strategies of document literacy and their conditions of use. Journal of Educational Psychology, 88, 314-332.

Ozuru, Y., Best, R., Bell, C., Witherspoon, A., \& McNamara, D. S. (2007). Influence of question format and text availability on assessment of expository texts comprehension. Cognition \& Instruction, 25, 399-438.

Pearson, P. D., Garvaglia, D. R., Lycke, K., Roberts, E., DanRIDGE, J., \& HAMM, D. (1999). The impact of item format on the depth of students' cognitive engagement (Tech. Rep.). Washington, DC: American Institutes for Research.

Pearson, P. D., \& Hamm, D. N. (2005). The assessment of reading comprehension: A review of practices-past, present, and future. In S. G. Paris \& S. A. Stahl (Eds.), Children's reading comprehension and assessment (pp. 13-69). Mahwah, NJ: Erlbaum.

Perfetti, C. (1985). Reading ability. New York: Oxford University Press.

Perfetti, C., Beck, I., Bell, L., \& Hughes, C. (1987). Phonemic knowledge and learning to read are reciprocal: A longitudinal study of first grade children. Merrill-Palmer Quarterly, 33, 283-319.

RICHTER, T. (2006). What is wrong with ANOVA and multiple regression? Analyzing sentence reading times with hierarchical linear models. Discourse Processes, 41, 221-250.

Scientific Software International (2007, March). HLM 6 for Windows. Retrieved March 15, 2007 from www.ssicentral.com/hlm/ student.

SheEHAN, K. M., \& Ginther, A. (2001, April). What do passage-based multiple-choice verbal reasoning items really measure? An analysis of the cognitive skills underlying performance on the current TOEFL reading section. Paper presented at the 2001 Annual Meeting of the National Council of Measurement in Education.

SNOw, C. E. (2003). Assessment of reading comprehension. In A. P. Sweet \& C. E. Snow (Eds.), Rethinking reading comprehension (pp. 192-218). New York: Guilford.

Snow, C. E., Barnes, W. S., Chandler, J., Goodman, I. F., \& HempHILL, L. (1991). Home and school influences on literacy. Cambridge, MA: Harvard University Press.

SteRnberG, R. J. (1991). Are we reading too much into reading comprehension tests? Journal of Reading, 34, 540-545.

Valencia, S. W., \& Pearson, P. D. (1988). Principles for classroom comprehension assessment. Remedial \& Special Education, 9, 26-35.

van den Broek, P., Lorch, R. F., Jr., Linderholm, T., \& GustafsON, M. (2001). The effects of readers' goals on inference generation and memory for texts. Memory \& Cognition, 29, 1081-1087.

VanderVeen, A., Huff, K., Gierl, M., McNamara, D. S., LouWerse, M., \& GRAESSER, A. (2007). Developing and validating instructionally relevant reading competency profiles measured by the critical reading section of the SAT. In D. S. McNamara (Ed.), Reading comprehension strategies: Theory, interventions, and technologies (pp. 137-172). Mahwah, NJ: Erlbaum.

Weiss, D. J., \& Kingsbury, G. G. (1984). Application of computerized adaptive testing to educational problems. Journal of Educational Measurement, 21, 361-375.

Wolfe, M. B., \& Goldman, S. R. (2005). Relationships between adolescents' text processing and reasoning. Cognition \& Instruction, 23, 467-502.

(Manuscript received September 27, 2007; revision accepted for publication March 26, 2008.) 